\title{
Interactions of small polypeptides with dimyristoylphosphatidylcholine monolayers: effect of size and hydrophobicity
}

\author{
N.A. Williams ${ }^{1}$ and N.D. Weiner ${ }^{2}$ \\ College of Pharmacy, University of Michigan, Ann Arbor, MI 48109 (U.S.A.)
}

Key words: Dimyristoylphosphatidylcholine monolayer; Polypeptide; Film penetration; Hydrophobicity

\begin{abstract}
Summary
The effects of size and hydrophobicity of small (molecular weights below 2,000) polypeptides on their predominantly hydrophobic interactions with a neutral phospholipid monolayer were studied. The changes in surface pressure were determined when various concentrations of Gly, Gly-Gly-Gly, L-Ala, L-Ala-L-Ala-L-Ala, L-Ala-Gly-Gly-Gly-Gly, L-Phe-L-Leu-L-Glu-L-Glu-L-Leu, adrenocorticotropic hormone fragments 1-10 (ACTH-(1-10), porcine $\beta$-lipotropin, $\alpha$-endorphin and human fibrinopeptide $A$ were injected under dimyristoylphosphatidylcholine (DMPC) monolayers at an initial surface pressure of $10 \mathrm{dyne} / \mathrm{cm}$. In all cases, when peptides with the same number of residues are compared, the concentration needed to increase the surface pressure of the film by 1 dyne $/ \mathrm{cm}$ was inversely related to its hydrophobicity. A reasonably good correlation was found to exist between the calculated free energy of transfer of a polypeptide from ethanol to water (a measure of its hydrophobicity) and its ability to increase the surface pressure of the DMPC film (a measure of the extent of its interaction with the neutral lipid monolayer).
\end{abstract}

The lipid bilayer: aqueous compartment partition coefficient of a liposomally encapsulated drug is a determining factor in its ability to enhance its in vitro and in vivo stability (Eppstein and Marsh, 1983) and its propensity to interact with and permeate biomembranes (Tremblay et al., 1984; Ganesan et al., 1984). Recent interest in the utilization of liposomally encapsulated polypeptides as a means of improving stability and bioavailability and providing rate-controlled delivery of these potent therapeutic agents (Eppstein and Marsh, 1983) necessitate the development of methodology to study their interactions with oriented arrays of

\footnotetext{
Present address: Liquid Products Department, Ahhott Laboratories, North Chicago, IL 60064, U.S.A.

Correspondence: N.D. Weiner, College of Pharmacy, University of Michigan, Ann Arbor, MI 48109, U.S.A.
}

lipids. Monolayer penetration experiments are a valuable tool for exploring interactions between lipid monolayers and proteins and can, with limitations, provide insight into protein-lipid bilayer interactions occurring in liposomal systems (Phillips et al., 1975; Phillips and Chapman, 1968; Kimelberg and Papahadjopoulos, 1971; Takada et al., 1982). Although penetration studies have helped elucidate the role of many factors, including protein hydrophobicity and flexibility, lipid compressibility and electrostatic interactions on the mechanism of lipid monolayer and membrane interactions with protein (Colacicco, 1970, 1972), such studies have invariably been performed with proteins or polypeptides having molecular weights above 5000 . Studies using smaller polypeptides have focussed on primarily electrostatic interactions with charged phospholipid monolayers (Takada et al., 1982). 
In this work, we have sought to investigate the effects of size and hydrophobicity of polypeptides having molecular weights below 2000 on their predominantly hydrophobic interactions with a neutral phospholipid monolayer, dimyristoylphosphatidylcholine (DMPC).

Gly, Gly-Gly-Gly (Peninsula Labs., Belmont, CA), L-Ala, L-Ala-L-Ala-L-Ala, L-Ala-GlyGly-Gly-Gly (Bachem, Inc., Torrance; CA), LPhe-L-Leu-L-Glu-L-Glu-L-Leu, adrenocorticotropic hormone fragments 1-10 (ACTH (1-10), L-Ser-L-Tyr-L-Ser-L-Met-L-Glu-L-His-L-Phe$\mathrm{L}$-Arg-L-Trp-Gly), porcine $\beta$-lipotropin ( $\mathrm{L}-$ Glu-L-Leu-L-Ala-Gly-L-Ala-L-Pro-L-Pro-LGlu-L-Pro-L-Ala), $\alpha$-endorphin (L-Tyr-GlyGly-L-Phe-L-Met-L-Thr-L-Ser-L-Glu-L-Lys-LSer-L-Glu-L-Thr-L-Pro-L-Leu-L-Val-L-Thr), human fibrinopeptide A (L-Ala-L-Asp-L-SerGly-L-Glu-Gly-L-Asp-L-Phe-L-Leu-L-Ala-LGlu-Gly-Gly-Gly-L-Val-L-Arg) and DMPC (Sigma, St. Louis, MO) were used as received. All other chemicals were of reagent grade and used as obtained.

The procedure used for the monolayer penetration studies was a modification of that reported by Schwinke et al. (1983). The experiments were performed at $25 \pm 1^{\circ} \mathrm{C}, \approx 2^{\circ} \mathrm{C}$ above the phase transition temperature of DMPC (Janiak et al., 1976). A Rosano surface tensiometer (Laboratory Products, Inc., Boston, MA) having a sensitivity of 0.05 dyne $/ \mathrm{cm}^{2}$, equipped with a sandblasted platinum Wilhelmy plate was used to measure surface tension. The subphase consisted of $90.0 \mathrm{ml}$ of $0.05 \mathrm{M}$ $N$-2-hydroxyethylpiperazine- $N$ '-2-ethane sulfonic acid (HEPES) at pH 7.0. Sodium chloride was added to adjust the ionic strength to 0.20 . The solution was contained in a circular polytetrafluorothylene dish $7.63 \mathrm{~cm}$ in internal diameter. A polytetrafluoroethylene-coated magnetic stirrer was used for mixing the subphase without disturbing the surface. The entire apparatus was enclosed to reduce airborne contamination.

DMPC was spread from a solution of $n$ hexane: ethanol $(9: 1, v / v)$ by means of an Agla micrometer syringe (Wellcome Reagents Ltd, Beckenham, U.K.) in amounts sufficient to produce the desired initial surface pressure. The addition of the spreading solvent alone to a clean buffer surface produced no measurable change in surface tension.

A stationary needle with a removable glass syringe was used to deliver concentrated peptide solutions beneath the surface into the subphase. An equal volume of the subphase was removed prior to the addition so as to keep the total volume constant. A magnetic stirrer $(\approx 80 \mathrm{rpm})$ mixed the subphase for $15 \mathrm{~min}$, and the system was allowed to equilibrate for $15 \mathrm{~min}$ before surface tension readings were recorded. Surface tension changes due to peptide addition were virtually complete within 30 min.

Surface pressure, $\pi$, was calculated as the difference in surface tension in the absence of the DMPC film and that of the film-covered surface. The change in surface pressure, $\Delta \pi$, was calculated as the difference in surface pressure of the lipid film upon injection of peptide into the subphase and that of the film in the absence of peptide, i.e., at its initial surface pressure. The surface tension of solutions of each of the peptides of all concentrations used in the penetration experiments was identical to that of the peptide-free buffer solution, indicating that the pressures measured in the presence of the DMPC monolayer were not caused by the peptides independently (at the concentrations employed) or by any impurities in them.

Preliminary experiments indicated that if the initial surface pressure was greater than 10 dyne $/ \mathrm{cm}$, injection of amino acids or tripeptides, even at high concentrations, did not show significant interactions with the monolayer as evidenced by changes in $\Delta \pi$ values. Therefore, 10 dyne $/ \mathrm{cm}$ was chosen as the optimal initial surface pressure for these studies.

Fig. 1 shows the change in surface pressure as a function of peptide "monomer" concentration at an initial pressure of $10 \mathrm{dyne} / \mathrm{cm}$. The term "monomer" concentration refers to the concentration of a single amino acid or the amino acid residue of a polypeptide. The monomer concentration was obtained by multiplying the molar concentration of the peptide by the number of amino acid units (whether similar or different) comprising it. For example, for L-Phe-L-Leu-L-Glu-LGlu-L-Leu, a $0.1 \mathrm{M}$ concentration of this poly- 

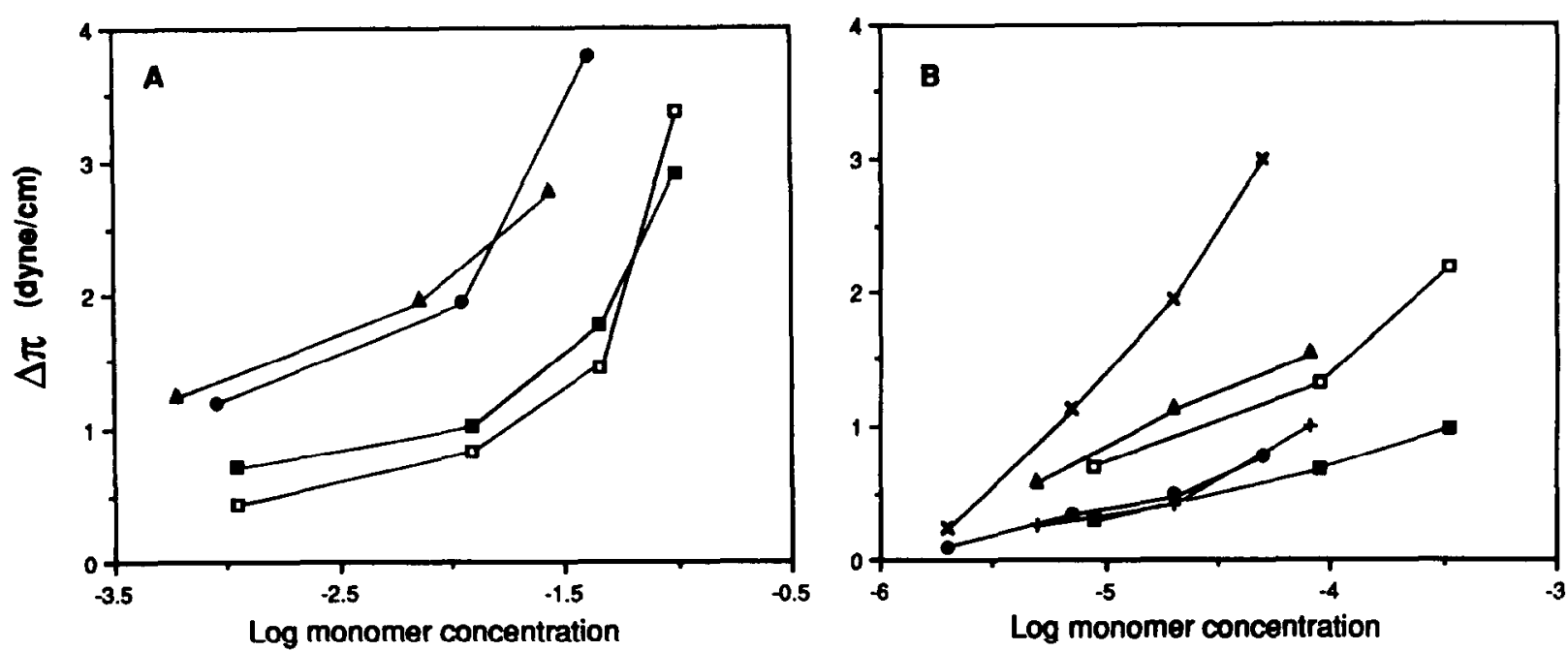

Fig. 1. Plots of the change in surface pressure as a function of peptide "monomer" concentration. The peptides were injected under a film of dimyristoylphosphatidylcholine at an initial pressure of $10 \mathrm{dyne} / \mathrm{cm}$. See text for experimental details. A: $\square$, Gly: Gly-Gly-Gly; 口, L-Ala; A, L-Ala-L-Ala-L-Ala; (B): 口, L-Ala-Gly-Gly-Gly-Gly; $\square$, L-Phe-L-Leu-L-Glu-L-Glu-L-Leu; X, ACTH-(1-10); $\uparrow$, porcine $\beta$-lipotropin, $\Delta, \alpha$-endorphin; + , human fibrinopeptide $A$.

peptide corresponds to a "monomer" concentration of $0.5 \mathrm{M}$. This approach enabled us to determine the relative extents of interactions of polypeptides with DMPC monolayers utilizing different concentrations of peptides of dissimilar amino acid sequences. The "monomer" concentration of each peptide required to increase the surface pressure 1 dyne $/ \mathrm{cm}$ (denoted as $C_{M}^{l}$ ) was obtained from the curves shown in Fig. 1 (short extrapolations of the Gly-Gly-Gly, L-Ala-L-Ala-L-Ala and porcine $\beta$-lipotropin curves were made). These values are shown in Table 1.

Also shown in Table 1 are the calculated values for the total free energy of transfer $\left(\Delta f_{t}\right)$ of the side-chains of each of the peptides from ethanol to water at $25^{\circ} \mathrm{C}$. These values, which represent relative indicators of hydrophobicity, were calculated from $\Delta f_{\mathrm{t}}$ values of individual amino acids reported by Nozaki and Tanford (1971). The more hydrophobic side chains will have a large positive $\Delta f_{\mathrm{t}}$ value and glycine as the reference is given a value of zero. Since it is generally accepted that hydrophobicities of amino acid residues are additive (Tanford, 1962; Bigelow, 1967; Segrest and Feldmann, 1974; Eisenberg, 1984), $\Delta f_{\mathrm{t}}$ for each peptide can be expressed either as the total free energy of transfer or as the free energy of transfer per amino acid residue.

Although several other approximations of hydrophobicities of amino acid residues have been reported (Eisenberg, 1984), Nozaki and Tanford's (1971) scale appears to us to be the most self-consistent. Their compilation omits several amino acid residues with charged side changes at $\mathrm{pH} 7$, i.e., glutamic acid, aspartic acid, arginine and lysine, because of the difficulty of measuring meaningful hydrophobicities for such side chains using ethanol as the non-polar phase. Ideally, the hydrophobicities of such side chains should be determined from solubilities of the amino acids in a pure hydrocarbon solvent (rather than the semi-polar ethanol) and water (Tanford, personal communication), but no such data are available. Since charged side chains are likely to remain in the aqueous phase and therefore contribute little to the surface pressure, we have assigned to them a value of zero. This type of approximation is not without precedent (Bigelow, 1967; Segrest and Feldmann, 1974). While this approximation may be appropriate for the present study where a neutral monolayer is present, it should be pointed out that in the presence of a charged monolayer, charged side chains 
TABLE 1

Relationships between the size and hydrophobicity of peptides and their interactions with dimyristoylphosphatidylcholine monolayers

\begin{tabular}{llccc}
\hline Peptide & $\begin{array}{l}\text { Number of } \\
\text { Residues }\end{array}$ & $\begin{array}{l}\text { Molecular } \\
\text { Weight }\end{array}$ & $\begin{array}{l}\text { Total } \Delta f_{\mathrm{t}} \\
\text { (Kcal/mole) }\end{array}$ & $\begin{array}{c}\text { Monomer" } \\
\text { Concn }\left(\mathrm{C}_{\mathrm{M}}^{1}\right)\end{array}$ \\
\hline glycine & 1 & 75.1 & 0 & $1.8 \times 10^{-2}$ \\
L-Alanine & 1 & 89.1 & 0.5 & $1.3 \times 10^{-2}$ \\
Gly-Gly-Gly & 3 & 189.2 & 0 & $5.6 \times 10^{-4}$ \\
L-Ala-L-Ala-L-Ala & 3 & 231.2 & 1.5 & $2.8 \times 10^{-4}$ \\
L-Ala-Gly-Gly-Gly-Gly & 5 & 317.3 & 0.5 & $2.5 \times 10^{-4}$ \\
L-Phe-L-Leu-L-Glu-L-Glu-L-Leu & 5 & 649.7 & 6.1 & $2.8 \times 10^{-5}$ \\
Porcine $\beta$-lipotropin & 10 & 951.0 & 7.8 & $7.9 \times 10^{-5}$ \\
ACTH-(1-10) & 10 & 1299.6 & 9.4 & $5.6 \times 10^{-6}$ \\
Human fibrinopeptide A & 16 & 1461.4 & 6.5 & $7.9 \times 10^{-5}$ \\
$\alpha$-Endorphin & 16 & 1745.9 & 12.3 & $1.6 \times 10^{-5}$ \\
\hline
\end{tabular}

are likely to influence strongly the surface-seeking tendency of a peptide. Therefore, when electrostatic interactions are present, the surface activity of an otherwise quite polar peptide may be either exaggerated or underestimated depending upon whether charge attraction or repulsion predominates. The advantages and limitations of utilizing the approach of Nozaki and Tanford and other approaches to describe polypeptide hydrophobicities have been discussed in detail (Eisenberg, 1984).

In all cases, when peptides with the same number of residues are compared, the more hydrophobic one exhibits a lower $C_{M}^{1}$ value (Table 1). An examination of Table 1 also shows that molecular weight (or size) influences the extent of interaction. For example, L-Ala-L-Ala-L-Ala, with roughly 3 times the molecular weight of L-Ala requires only $1 / 45$ the equivalent concentration of the monomer to cause the same change in surface pressure. A similar effect of chain length has been demonstrated for the interaction of polylysine and lysine with a negatively charged polyphosphoinositide monolayer (Takada et al., 1982).

An examination of Fig, 2 reveals that a reasonably good correlation exists between the free energy of transfer of a polypeptide from ethanol to water (a measure of its hydrophobicity) and its $C_{M}^{1}$ value (a measure of the extent of its interaction with the neutral lipid monolayer). Since both the size of the polypeptide and the hydrophobicity of its individual amino acid residues affect its interaction with the DMPC monolayer, the total free energies of transfer values rather than the $\Delta f_{t}$ per residue were used. It should be pointed out that calculations of this type do not account for the possibility that secondary structure may be present in the larger polypeptides studied, and that such structures would be altered upon interaction with the monolayers.

It is well known that proteins most readily penetrate phospholipid monolayers when the film

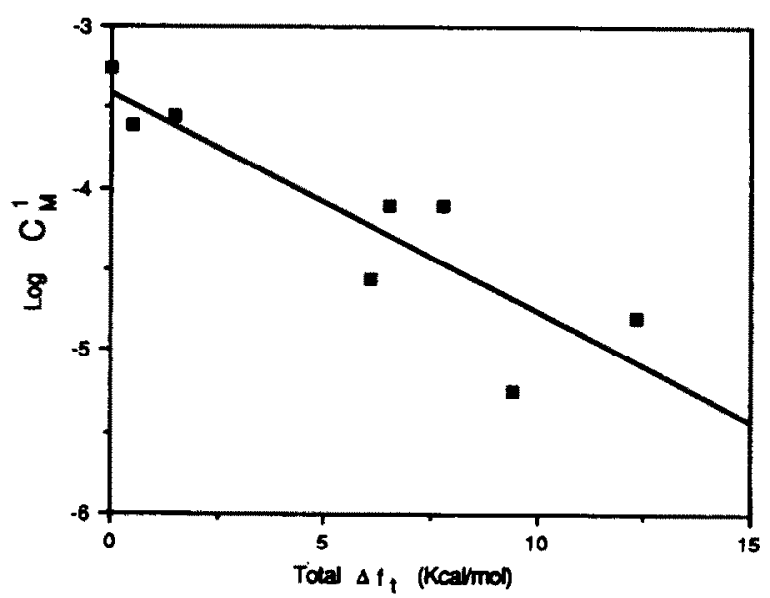

Fig. 2. Plot of the peptide "monomer" concentration required to increase the surface pressure of a DMPC monolayer 1 dyne/cm ( $C_{M}^{1}$; a measure of the extent of its interaction with the neutral lipid monolayer) vs its free energy of transfer from ethanol to water $\left(\Delta f_{i}\right.$; a measure of its hydrophobicity). $C_{M}^{l}$ values were obtained from the curves shown in Fig. 1. See text for experimental details. 
pressure is low (Quinn and Chapman, 1980; Van Deenen et al., 1976). As the lipid film is condensed, closer packing of the lipid molecules will tend to exclude the protein from an area-determining position. However, when conditions are optimal, e.g., surface active proteins interacting with charged lipid films, film penetration is evident at surface pressures as high as 60 dyne $/ \mathrm{cm}$ (Hendrickson et al., 1983). Even in the absence of primary electrostatic interactions, proteins have a capacity to interact hydrophobically with neutral phospholipid monolayers resulting in film penetration still seen at surface pressures comparable to the internal lateral pressures expected in phospholipid bilayers (Hendrickson et al., 1983; Pattus et al., 1979; Lea et al., 1975; Pink, 1984).

Since the molecular packing and lateral pressures of the monolayer and bilayer system are virtually the same at the equilibrium surface tension of liposomes (MacDonald and Simon, 1987), it is not surprising that results obtained from monolayer penetration studies have been successful in furthering our understanding of how peptides affect phospholipid bilayer systems in natural membranes (Bougis et al., 1981) and liposomes (Teissie, 1981). The partitioning of a peptide between the lipid bilayers and aqueous compartments of a liposome is very difficult to measure experimentally and is not easily predicted since its value would be quite dependent on specific and non-specific intermolecular interactions occurring between the peptide and the oriented lipid array. Thus, even highly charged, very water-soluble compounds can become associated with lipid bilayers via predominantly specific interactions ( $\mathrm{Au}$ et al., 1986). Since the partition coefficient of a bioactive peptide within a liposome would be expected to influence its stability and therapeutic efficacy (Eppstein and Marsh, 1983), experimental procedures to determine the effects of lipid and peptide structure on their propensity to interact with each other would be most useful. The correlation shown in Fig. 2, which can in principle also be done at higher surface pressures, e.g., 30-40 dyne $/ \mathrm{cm}$, suggests that relative accumulation of polypeptides at oriented lipid interfaces can be estimated by penetration experiments of this type. It remains to be seen whether these subtle hydro- phobic effects will be apparent with charged monolayers, where electrostatic interactions predominate, and whether data of this type can be extrapolated to explain the distribution of small polypeptides in liposomes.

\section{Acknowledgements}

This study was supported by grant $\mathrm{AI} / \mathrm{GM}$ 22303 from the National Institutes of Health.

\section{References}

Au, S., Weiner, N. and Schacht, J., Membrane perturbation by aminoglycosides as a simple screen of their toxicity. Antimicrob. Agents Chemother., 30 (1986) 395-397.

Bigelow, C.C., On the average hydrophobicity of proteins and the relation between it and protein structure. J. Theor. Biol., 16 (1967) 187-211.

Bougis, P., Rochat, H., Pieroni, G. and Verger, R., Penetration of phospholipid monolayers by cardiotoxins. Biochemistry, 20 (1981) 4915-4920.

Colacicco, G., Lipid monolayers: mechanisms of protein penetration with regard to membrane models. Lipids, 636 (1970) 636-649.

Colacicco, G., Surface behavior of membrane proteins. N.Y. Acad. Sci., 195 (1972) 224-261.

Eisenberg, D., Three-dimensional structure of membrane and surface proteins. Ann. Rev. Biochem., 53 (1984) 595-623.

Eppstein, D.A. and Marsh, Y.V., Partial dissociation of antiviral and antimitogenic activities of murine interferon after its incorporation into liposomes. J. Interferon Res., 3 (1983) 161-168.

Ganesan, M.G., Weiner, N.D., Flynn, G.L. and Ho, N.F.H., Influence of liposomal drug entrapment on percutaneous absorption. Int. J. Pharm., 20 (1984) 139-154.

Hendrickson, H.S., Fan, P.C., Kaufman, D.K. and Kleiner, D.E., The effect of a phase transition on penetration of phospholipid monolayers by melittin and glucagon. Arch. Biochem. Biophys., 227 (1983) 242-247.

Janiak, M.J., Small, D.M. and Shipley, G.G., Nature of the thermal pretransition of synthetic phospholipids: dimyristoyl- and dipalmitoyllecithin. Biochemistry, 15 (1976) 4575-4580.

Kimelberg, H.K. and Papahadjopoulos, D., Phospholipid-protein interactions: membrane permeability correlated with monolayer “penetration”. Biochim. Biophys. Acta, 233 (1971) 805-809.

Lea, E.J.A., Rich, G.T. and Segrest, J.P., The effects of the membrane-penetrating polypeptide segment of the human erythrocyte $\mathrm{MN}$-glycoprotein on the permeability of model lipid membranes. Biochim. Biophys. Acta, 382 (1975) 41-50. 
MacDonald, R.C. and Simon, S.A., Lipid monolayer states and their relationships to bilayers. Proc. Natl. Acad. Sci. U.S.A., 84 (1987) 4089-4093.

Nozaki, Y. and Tanford, C., The solubility of amino acids and two glycine peptides in aqueous ethanol and dioxane solutions. Establishment of a hydrophobicity scale. J. Biol. Chem., 246 (1971) 2211-2217.

Pattus, F., Slotboom, A.J. and de Haas, G.H., Regulation of phospholipase A2 activity by the lipid-water interface: a monolayer approach. Biochemistry, 18 (1979) 2691-2697.

Phillips, M.C. and Chapman, D., Monolayer characteristics of saturated 1,2,-diacyl phosphatidylcholines (lecithins) and phosphatidylethanolamines at the air-water interface. Biochim. Biophys. Acta, 163 (1968) 301-313.

Phillips, M.C., Hauser, H., Leslie, R.B. and Oldani, D., A comparison of the interfacial interactions of the apoprotein from high density lipoprotein and beta-casein with phospholipids. Biochim. Biophys. Acta, 406 (1975) 402-414.

Pink, D.A., Theoretical studies of phospholipid bilayers and monolayers. Perturbing probes, monolayer phase transitions, and computer simulations of lipid-protein bilayers. Can. J. Biochem. Cell. Biol., 62 (1984) 760-777.

Quinn, P.J. and Chapman, D., The dynamics of membrane structure. CRC Crit. Rev. Biochem., 8 (1980) 1-117.

Schwinke, D.L., Ganesan, M.G. and Weiner, N.D., Monolayer studies of insulin-lipid interactions. J. Pharm. Sci., 72 (1983) 244-248.
Segrest, J.P. and Feldmann, R.J., Membrane proteins: amino acid sequence and membrane penetration. J. Mol. Biol., 87 (1974) 853-858.

Takada, A., Lodhi, S., Weiner, N.D. and Schacht, J., Lysine and polylysine: correlation of their, effects on polyphosphoinositides in vitro with their ototoxic action in vivo. $J$. Pharm. Sci., 71 (1982) 1410-1411.

Tanford, C., Contribution of hydrophobic interactions to the stability of globular conformation of proteins. J. Am. Chem. Soc., 84 (1962) 4240-4247.

Teissie, J., Interaction of cytochrome $c$ with phospholipid monolayers. Orientation and penetration of protein as functions of the packing density of film, nature of the phospholipids, and ionic content of the aqueous phase. Biochemistry, 20 (1,981) 1554-1560.

Tremblay, C., Barza, M., Fiore, C. and Szoka, F., Efficacy of liposome-intercalated amphotericin B in the treatment of systemic candidiasis in mice. Antimicrob. Agents Chemother., 26 (1984) 170-173.

Van Deenen, L.L.M., Demel, R.A., Geurts van Kessel, W.S.M., Kamp, H.H., Roelofsen, B., Verkleij, A.J., Wirtz, K.W. and Zwaal, R.F.A., Phospholipases and monolayers as tools in studies of membrane structure. In: The Structural Basis of Membrane Function, Academic, New York, NY, 1976, p. 21. 